\title{
AVALIAÇÃO DA QUALIDADE DE VIDA DE HIPERTENSOS USUÁRIOS DE UMA UNIDADE BÁSICA DE SAÚDE
}

\author{
ASSESSMENT OF QUALITY OF LIFE OF HYPERTENSIVE USERS OF A HEALTH CARE CENTER
}

\author{
Ana Paula Kruger ${ }^{\mathrm{a}^{*}}$, Michelle Mergener ${ }^{\mathrm{b}^{*}}$, Adriane Pozzobon ${ }^{\mathrm{c}^{*}}$, Thaís Rodrigues Moreira ${ }^{\mathrm{d}^{*}}$ \\ aapk@universo.univates.br, bmichimerg@yahoo.com, cadripozz@yahoo.com.br, dth_rodrigues@ibest.com.br \\ *Centro Universitário Univates - Lajeado (RS), Brasil
}

Data de recebimento do artigo: 06/10/2014 Data de aceite do artigo: 08/05/2015

\section{RESUMO}

Introduçáo: A hipertensão arterial sistêmica é uma das patologias mais prevalentes no mundo, atingindo cerca de $30 \%$ da população adulta mundial. Dentre os fatores que elevam a pressão arterial destacam-se a obesidade, estresse e alimentaçáo inadequada. Objetivos: $\mathrm{O}$ objetivo deste estudo foi avaliar a qualidade de vida e verificar sua correlaçáo com a avaliaçáo antropométrica de pacientes hipertensos. Materiais e Métodos: Avaliaram-se 51 pacientes, por meio da aferição do peso corpóreo, altura, índice de massa corporal, circunferência da cintura, prega cutânea triciptal, circunferência muscular do braço, aferição da pressão arterial e aplicação do questionário para avaliação da qualidade de vida WHOQOL-bref. Resultados: Por meio da avaliação do índice de massa corporal, 53\% ( $\mathrm{n}=27)$ apresentaram excesso de peso, 45\% $(\mathrm{n}=23)$ eutrofia e $2 \%(n=1)$ magreza. Para a circunferência muscular do braço, $82 \%(n=42)$ eram eutróficos, sobre circunferência da cintura, $96 \%(\mathrm{n}=49)$ estavam com risco de desenvolvimento de doenças cardiovasculares e $90 \%(n=46)$ dos pacientes estavam com a pressão arterial elevada. Na avaliação do WHOQOL-bref, observou-se que quanto maiores as idades dos indivíduos, piores foram as percepçôes com relação aos domínios referentes ao meio ambiente e à qualidade de vida total. $\mathrm{O}$ escore geral de qualidade de vida do grupo não idoso foi significativamente superior ao do grupo idoso. Conclusóes: A maioria dos indivíduos da amostra apresentaram risco de desenvolvimento de doenças cardiovasculares e excesso de peso. Podese constatar que os indivíduos avaliados não idosos têm uma percepção melhor da qualidade de vida, apresentando um escore maior no questionário WHOQOL-bref quando comparados aos indivíduos idosos.

Palavras-chave: Hipertensão arterial; qualidade de vida; índice massa corporal.

\section{ABSTRACT}

Introduction: Hypertension is one of the most prevalent diseases in the world, reaching about $30 \%$ of adults worldwide. Among the factors that increase blood pressure stand out obesity, stress and inadequate nutrition. Objectives: The aim of this study was to evaluate the quality of life and to assess its correlation with the anthropometric assessment of hypertensive patients. Materials and Methods: We evaluated 51 patients by measuring their of body weight, height, body mass index, waist circumference, triceps skinfold thickness, arm muscle circumference, blood pressure measurement and application of the WHOQOL-bref questionnaire to assess the quality of life. Results: Through the body mass index, 53\% $(\mathrm{n}=27)$ were overweight, $45 \%(\mathrm{n}=23)$ normal weight and $2 \%(\mathrm{n}=1)$ thinness. For the arm muscle circumference, $82 \%(\mathrm{n}=42)$ were considered normal, and on waist circumference, $96 \%(\mathrm{n}=49)$ were at risk of developing cardiovascular disease, and $90 \%(\mathrm{n}=46)$ of patients had high blood pressure. In assessing the WHOQOL-bref, it was observed that the greater the age of individuals, the worst were the perceptions regarding areas relating to the environment and overall quality of life. The overall score for quality of life of the non-elderly group was not significantly higher than in the older group. Conclusions: The majority of individuals in the sample have risk of developing cardiovascular diseases and overweight. It can be seen that the assessed non-elderly individuals have a better perception of quality of life, with a higher score in the WHOQOL-bref questionnaire when compared to older people.

Keywords: Hypertension; quality of life; body mass index. 


\section{Introdução}

A hipertensão arterial sistêmica (HAS), conforme a VI Diretrizes Brasileiras de Hipertensão, é uma das patologias mais prevalentes no mundo, acometendo cerca de $30 \%$ da populaçáo adulta mundial ${ }^{1}$. Em contraponto, sabe-se que $12 \%$ da população adulta é obesa em nível mundial, e no período entre 1980 a 2008, essa prevalência foi duplicada ${ }^{2}$. Ademais, a obesidade é considerada um dos principais fatores de risco, independentemente da faixa etária, para o desenvolvimento da $\mathrm{HAS}^{3}$.

O desenvolvimento da HAS contribui diretamente para o aumento da morbimortalidade causada por doenças cardiovasculares (DCV), pois quando estas não são fatais, levam com frequência à invalidez parcial ou total, com graves consequências para o indivíduo ${ }^{4}$. Ressalta-se ainda que o indivíduo hipertenso tem até sete vezes mais risco de acidente vascular cerebral, triplicando o risco de infarto do miocárdio - a causa mais comum de insuficiência cardíaca 5 .

Diversos fatores são considerados contribuintes para a alteração dos níveis pressóricos, podendo-se destacar o estresse, esforço físico excessivo, etilismo e tabagismo ${ }^{6}$. Cabe salientar que o aparecimento da HAS é favorecido pelo excesso de peso, sedentarismo, baixa ingestáo de potássio, dislipidemias, intolerância à glicose, diabetes mellitus e menopausa ${ }^{7}$.

A mudança do perfil de morbimortalidade, tendência universal também nos países em desenvolvimento, indica o aumento da prevalência das doenças crônico-degenerativas. Os avanços nos tratamentos e as possibilidades efetivas de controle dessas enfermidades têm acarretado o aumento da sobrevida e/ou a vida longa das pessoas acometidas por esses agravos. Assim, mesmo que o objetivo principal do tratamento da HAS seja a normalização dos níveis da pressão arterial (PA), por meio da adoção de hábitos dietéticos saudáveis, evitando alimentos ricos em sódio, e incorporando a prática regular de atividade física e a manutenção de peso corpóreo, são adotadas medidas terapêuticas que envolvem principalmente mudanças no estilo de vida, visando diminuir a interferência dos fatores de risco. Desta forma, o objetivo do serviço de saúde, que era o de remediar a patologia, ganhou um novo rumo, a prevenção e o controle dos fatores de risco, tornando a qualidade de vida $(\mathrm{QV})$ e da alimentação dos indivíduos hipertensos foco relevante no tratamento e prevenção da $\mathrm{HAS}^{1,4}$.

$\mathrm{Na}$ área da saúde, a QV sofre a influência dos determinantes e condicionantes do processo saúde-doença, que são multifatoriais e complexos. Dessa forma, saúde e doença estão relacionadas aos aspectos econômicos, socioculturais, à experiência pessoal e estilos de vida. A melhoria da QV pode ser considerada como um dos resultados esperados, tanto das práticas assistenciais quanto das políticas públicas para o setor nos campos da promoção da saúde e da prevenção de doenças. Além disso, informaçóes sobre QV têm sido incluídas tanto como indicadores para avaliação da eficácia, eficiência e impacto de determinados tratamentos para grupos de portadores de agravos diversos, quanto na comparaçáo entre procedimentos para o controle de problemas de saúde ${ }^{4}$.

Devido à crescente importância da avaliação dos fatores interferentes na QV, pelas diferentes áreas da saúde, foi desenvolvido um instrumento de avaliação de qualidade de vida com 100 questôes, o World Health Organization Quality of Life - WHOQOL ${ }^{8}$. Porém, a necessidade de instrumentos curtos que demandassem pouco tempo para seu preenchimento, fez com que fosse desenvolvido o instrumento WHOQOL-bref com abrangência dos quatro domínios: físico, psicológico, relaçôes sociais e meio ambiente?

Considerando o caráter multifatorial para o desenvolvimento da HAS, este estudo objetivou avaliar a QV e verificar sua correlação com os parâmetros antropométricos de indivíduos hipertensos, a fim de sugerir fatores que mereceriam maior atenção no tratamento ou na prevenção da HAS entre a população.

\section{Metodologia}

Trata-se de um estudo transversal com amostra composta por indivíduos adultos e idosos, de ambos os gêneros e frequentadores do grupo Hiperdia, de uma Unidade Básica de Saúde (UBS), de um município do interior do Rio Grande do Sul. Foram excluídos do estudo indivíduos com diagnóstico de cardiopatias e em tratamento farmacológico destas, bem como os que não estavam presentes no dia da coleta de dados.

O estudo obteve aprovação do Comitê de Ética em Pesquisa do Centro Universitário Univates, sob o protocolo de $\mathrm{n}^{\circ}$ 122/11. A coleta dos dados foi realizada no período de março a junho de 2012, no qual foi realizado o recrutamento dos participantes e a coleta de dados nas dependências da UBS. Inicialmente realizou-se a explicaçáo do estudo e seus objetivos; em seguida, os indivíduos foram convidados a participar de forma voluntária e foi entregue o termo de consentimento livre e esclarecido.

A avaliação antropométrica foi composta pela aferição de peso, estatura, índice de massa corporal (IMC), circunferência da cintura (CC), dobra cutânea triciptal (DCT) e circunferência muscular do braço (CMB). Para tal, os indivíduos foram orientados a ficar descalços e com roupas leves. Aferiçáo do peso foi realizada com a utilização de balança eletrônica da marca Plenna ${ }^{\oplus}$, modelo Giant Lithium, com capacidade para $150 \mathrm{~kg}$ e 
precisão de 100 gramas. Após, para aferição da altura, o paciente foi posicionado de costas para uma parede lisa e sem rodapé e com a cabeça reta. Foi utilizado o estadiômetro portátil da marca $\mathrm{Seca}^{\oplus}$, com precisão de $0,1 \mathrm{~cm}$.

A mensuraçáo da CC foi realizada com o indivíduo em posiçấo ortostática, colocando-se sobre a pele uma fita inelástica graduada em milímetros, no espaço correspondente à menor circunferência entre a crista ilíaca e o rebordo costal ${ }^{10}$. Classificaçáo desta foi conforme os seguintes parâmetros: homens $>94 \mathrm{~cm}$ e mulheres $>80$ $\mathrm{cm}$ foram considerados com risco cardiovascular ${ }^{11}$.

Aferiu-se a circunferência do braço com este solto e relaxado, após circundando-se com auxílio de fita métrica inelástica, o ponto médio entre o processo acromial da escápula e o olécrano ${ }^{12}$. E nesta mesma localização foi marcado o ponto médio e, aproximadamente dois centímetros acima deste ponto, a DCT foi pinçada entre o polegar e o indicador; em seguida, foi lida a medida que o adipômetro clínico acusou ${ }^{13}$. A classificação da DCT foi realizada por meio da adequação do percentil 50, seguindo os seguintes parâmetros: desnutrição grave $<70 \%$, desnutrição moderada $70-80 \%$, desnutrição leve $80-90 \%$, eutrofia $90-110 \%$, sobrepeso $110-120 \%$ e obesidade $>120 \%{ }^{12}$. Ainda, a CBM foi calculada e classificada conforme os parâmetros estabelecidos por Blackburn e Thornton ${ }^{12}$.

Por meio dos dados de peso e altura, calculou-se o IMC, e este foi classificado conforme os parâmetros para adultos estabelecidos pela World Health Organization $(\mathrm{WHO})^{14}$. E os seguintes parâmetros foram utilizados para idosos ${ }^{15}$ : magreza $<22 \mathrm{~kg} / \mathrm{m}^{2}$, eutrofia $22-27 \mathrm{~kg} / \mathrm{m}^{2}$ e excesso de peso $<27 \mathrm{~kg} / \mathrm{m}^{2}$.

Os níveis de PA foram aferidos por um enfermeiro por meio do método indireto, utilizando um esfigmomanômetro aneróide, periodicamente testado e devidamente calibrado. Certificando-se de que o paciente não tinha praticado exercícios físicos, não tinha ingerido bebidas alcoólicas, café, alimentos ou fumado até 30 minutos antes da aferição, a medida da PA foi realizada na posiçáo sentada, com o braço direito apoiado sobre uma mesa, à altura do coração. A artéria braquial foi localizada pela palpação e o manguito foi colocado firmemente centralizando a bolsa de borracha sobre a artéria braquial. A pressão sistólica foi aferida no momento do aparecimento do primeiro som (fase I de Korotkoff) e a determinaçáo da pressáo diastólica se deu por meio do desaparecimento do som (fase $\mathrm{V}$ de Korotkoff). Em seguida procedeu-se com a desinsuflação rápida e completa $^{16}$. A classificação foi utilizada considerando-se os valores de PA ótima: $<120 \mathrm{x}<80$, normal: $<130 \mathrm{x}<85$, hipertensão estágio 1: 140x90, hipertensão estágio 2: 160x100, hipertensão estágio 3: $>180 x>110^{1}$.

Por fim, foi explicado e aplicado individualmente pela pesquisadora o questionário WHOQOL-bref, composto por 26 questóes que abrangem os quatro domínios: físico, psicológico, relaçôes sociais e meio ambiente. As questóes oferecem cinco pontos de resposta de acordo com a escala de Likert, um tipo de escala de resposta psicométrica usada habitualmente em questionários, principalmente em pesquisas de opinião. Ao responderem tal questionário, os perguntados especificam seu nível de concordância com uma afirmação. Após o questionário ter sido totalmente respondido, cada item pôde ser analisado separadamente, mas no caso deste estudo, optou-se por analisar a soma das respostas dadas, a fim de criar um resultado por escore. Tal escore é apresentado numa faixa de zero a 100 , onde o escore zero indica o pior nível e 100 o melhor nível de qualidade de vida ${ }^{9}$.

Para a análise estatística utilizou-se o teste $\mathrm{t}$ de Student para a comparaçáo dos resultados dos escores do WHOQOL-bref entre os gêneros, a classificação de idoso e não idoso e a análise de correlaçáo de Pearson para correlacionar os escores do WHOQOL-bref com outras variáveis quantitativas do estudo. Para todos os testes, o nível de significância máximo assumido foi de $5 \%(\mathrm{p} \leq 0,05)$ e o software utilizado para a análise estatística foi o Statistical Package for the Social Sciences (SPSS), na versão 17.0.

\section{Resultados}

Foram avaliados 51 indivíduos hipertensos, dentre estes $64,7 \%(n=33)$ da amostra eram do gênero feminino. A média de idade dos indivíduos foi 59,5 $\pm 8,4$ anos, o peso médio de $74 \mathrm{~kg}$ e o IMC médio de $27 \mathrm{~kg} / \mathrm{m}^{2}$. $\mathrm{Na}$ Tabela 1, pode-se verificar pela avaliação da CMB, $82,4 \%(\mathrm{n}=42)$ eram eutróficos, porém na medida da CC, 96,1\% ( $\mathrm{n}=49)$ dos indivíduos avaliados apresentaram valores mais elevados que aqueles considerados adequados. Constatou-se que a maior parte da amostra apresentava risco para o desenvolvimento de $\mathrm{DCV}$, conforme a classificação da CC.

Constatou-se que 46 indivíduos estavam com a PA elevada, apresentando valores superiores a 140/90 $\mathrm{mmHg}$. O tratamento farmacológico foi a medida de controle da HAS utilizada por $90,20 \%(n=46)$ dos indivíduos e os demais não utilizavam esse recurso terapêutico. Apenas cinco indivíduos apresentaram o nível de PA considerado adequado.

$\mathrm{Na}$ Tabela 2, observou-se correlaçáo positiva dos escores para o domínio meio ambiente total, onde foram maiores os valores para CMB ( $r=0,293)$, e correlação negativa, onde menores foram os escores para os domínios de meio ambiente $(\mathrm{r}=-0,369)$ e qualidade de vida total $(\mathrm{r}=-0,437)$. 
As comparaçóes entre os domínios e os gêneros encontram-se na Tabela 3, sendo que os resultados dos domínios que demonstraram diferença significativa entre os gêneros foram: psicológico total $(\mathrm{p}=0,040)$ e meio ambiente total $(\mathrm{p}=0,040)$, indicando que, em ambos os domínios, as mulheres apresentaram uma percepção

Tabela 1: Classificação nutricional de indivíduos hipertensos.

\begin{tabular}{|c|c|c|c|}
\hline Variável & Classificaçáo & $\mathbf{N}$ & $\%$ \\
\hline \multirow[t]{3}{*}{ IMC } & Magreza & 1 & 2,0 \\
\hline & Eutrofia & 23 & 45,1 \\
\hline & Excesso de peso & 27 & 52,9 \\
\hline \multirow[t]{6}{*}{ DCT } & Desnutrição grave & 4 & 7,8 \\
\hline & Desnutrição moderada & 4 & 7,8 \\
\hline & Desnutrição leve & 3 & 5,9 \\
\hline & Eutrofia & 14 & 27,5 \\
\hline & Sobrepeso & 4 & 7,8 \\
\hline & Obesidade & 22 & 43,1 \\
\hline \multirow[t]{4}{*}{ CMB } & Desnutrição grave & 2 & 3,9 \\
\hline & Desnutrição moderada & 2 & 3,9 \\
\hline & Desnutrição leve & 5 & 9,8 \\
\hline & Eutrofia & 42 & 82,4 \\
\hline \multirow[t]{2}{*}{ Risco CC } & Com risco & 49 & 96,1 \\
\hline & Sem risco & 2 & 3,9 \\
\hline
\end{tabular}

CC: circunferência da cintura; CMB: circunferência muscular do braço; DCT: dobra cutânea triciptal; IMC: índice de massa corporal.

Tabela 2: Relação dos domínios com a idade e o estado nutricional de indivíduos hipertensos.

\begin{tabular}{lcccccc}
\hline \multirow{2}{*}{ Domínios totais } & \multicolumn{5}{c}{ Coeficiente de Correlaçáo de Pearson (r) } \\
\cline { 2 - 7 } & IMC & CB & DCT & CMB & CC & Idade \\
\hline Físico & $-0,175$ & $-0,226$ & $-0,212$ & $-0,154$ & $-0,101$ & $-0,193$ \\
Psicológico & $-0,077$ & $-0,040$ & $-0,138$ & 0,044 & $-0,168$ & $-0,137$ \\
Relaçóes pessoais & 0,081 & 0,058 & $-0,042$ & 0,108 & $-0,008$ & $-0,225$ \\
Meio ambiente & 0,060 & 0,201 & $-0,031$ & $0,293^{*}$ & $-0,054$ & $-0,369^{* *}$ \\
Qualidade de vida & $-0,103$ & 0,015 & 0,029 & $-0,001$ & $-0,202$ & $-0,437^{* *}$ \\
\hline
\end{tabular}

IMC: índice de massa corporal; CB: circunferência do braço; DCT: dobra cutânea triciptal; CMB: circunferência muscular do braço; CC: circunferência da cintura.

* Significativo $\mathrm{p} \leq 0,05$;

** Significativo $\mathrm{p} \leq 0,01$.

Tabela 3: Comparação entre os domínios e os gêneros de indivíduos hipertensos.

\begin{tabular}{lccc}
\hline Domínios totais & Gênero & Média \pm desvio padrão & $\mathbf{p}^{*}$ \\
\hline Físico & Feminino & $58,8 \pm 8,7$ & 0,103 \\
& Masculino & $62,7 \pm 6,8$ & 0,040 \\
Psicológico & Feminino & $58,3 \pm 9,8$ & 0,118 \\
& Masculino & $63,4 \pm 7,2$ & 0,040 \\
Relaçóes pessoais & Feminino & $72,7 \pm 11,1$ & $0,37,3 \pm 6,9$ \\
Meio ambiente & Masculino & $65,7 \pm 9,3$ & 0,352 \\
Qualidade de vida & Feminino & $70,0 \pm 5,2$ & $70,1 \pm 14,3$ \\
& Masculino & $73,6 \pm 9,5$ & 0 \\
\hline
\end{tabular}

*Teste t de Student. Consideradas estatisticamente significativas as diferenças em que $\mathrm{p} \leq 0,05$. 
Tabela 4: Comparação entre os domínios e a idade de indivíduos hipertensos.

\begin{tabular}{lccc}
\hline Domínios totais & Idade & Média \pm desvio padrăo & $\mathbf{p}^{*}$ \\
\hline Físico & Idoso & $59,6 \pm 10,2$ & 0,708 \\
Psicológico & Não idoso & $60,5 \pm 6,9$ & 0,777 \\
& Idoso & $59,6 \pm 11,5$ & $60,4 \pm 7,8$ \\
Relaçóes pessoais & Não idoso & $73,2 \pm 11,0$ & 0,549 \\
Meio ambiente & Idoso & $75,0 \pm 9,5$ & 0,068 \\
& Não idoso & $64,5 \pm 10,8$ & 0,016 \\
\hline
\end{tabular}

*Teste t de Student. Consideradas estatisticamente significativas as diferenças em que $\mathrm{p} \leq 0,05$.

domínio avaliado. Os demais dados estão apresentados na Tabela 4.

\section{Discussão}

O excesso de peso está diretamente associado a alteraçóes estruturais e funcionais do sistema cardiovascular, pois ocasiona um aumento do volume sanguíneo e do débito cardíaco, contribuindo para a HAS, hipertrofia miocárdica e diminuição da complacência arterial ${ }^{17}$. A alta prevalência de sobrepeso e obesidade observada neste estudo, como também valores aumentados de circunferência da cintura, pode estar relacionada diretamente com tais fenômenos. Conforme a WHO, o aumento na prevalência da HAS ocorre em conjunto com o aumento do sobrepeso e obesidade na população ${ }^{2,14}$. O Framingham Heart Study sugere que 75\% dos homens e $65 \%$ das mulheres apresentaram HAS diretamente associada ao sobrepeso e obesidade ${ }^{18}$.

A literatura científica tem apresentado fortes evidências de que o IMC apresenta consistente relação com a gordura corporal total. A prevalência de sobrepeso e obesidade em indivíduos hipertensos também foi observada em diversos estudos ${ }^{19-22}$. Contudo, estudos científicos que avaliam a DCT em indivíduos hipertensos são escassos. Neste estudo foram verificados elevados índices de DCT nos indivíduos com sobrepeso e obesidade.

Em um estudo, realizado por Carneiro et al., demonstrou-se que indivíduos com idade entre 30 e 65 anos e com IMC elevado possuem maiores riscos de serem hipertensos em relação aos indivíduos eutróficos. E a prevalência de hipertensos no grupo com sobrepeso foi de $23 \%$ e nos obesos de grau III foi de $67,1 \%{ }^{23}$. Já em outro estudo, foi observada uma prevalência de $75,2 \%$ de hipertensos com excesso de peso e obesidadde $^{21}$. Esses trabalhos confirmam os resultados obtidos nesta análise.
Em relação a CC, neste estudo observou-se o aumento do risco de desenvolvimento de DCV na maioria dos indivíduos avaliados (as mulheres apresentaram maiores valores de CC). Dados semelhantes a estes foram verificados pelo estudo de Barbosa et al., que constataram que a CC manteve-se 2,4 vezes maior em indivíduos que apresentaram diagnóstico de HAS, quando comparados a valores de CC adequados em indivíduos saudáveis ${ }^{24}$. De forma semelhante, o estudo sobre risco cardiovascular realizado por Souza et al., baseado na avaliação do IMC e CC, observou que $50 \%$ da amostra apresentava elevado risco cardiovascular - tal resultado sugere que a obesidade, principalmente a abdominal, aumenta as chances de complicaçóes e $\mathrm{DCV}^{25}$. Resultados semelhantes a esse também foram observados por Lima et al., onde $75 \%$ dos indivíduos do gênero feminino apresentaram elevado risco para o desenvolvimento de DCV, através da análise e classificação da $\mathrm{CC}^{26}$.

Quanto ao controle da PA, no estudo de Melchiors et al., verificou-se que $69,6 \%$ dos pacientes com PA não controlada realizavam tratamento anti-hipertensivo, resultado próximo ao descrito no documento da $\mathrm{WHO}$ para HAS, no qual consta que três quartos dos pacientes hipertensos não alcançam o controle positivo da $\mathrm{PA}^{21}$. Neste trabalho pode-se constatar que a maioria da amostra utilizava fármacos anti-hipertensivos, porém, no momento da coleta, a maioria dos indivíduos apresentaram alteraçóes nos níveis pressóricos. Reforçando os dados apresentados, outro estudo observou que o controle da PA não está relacionado apenas ao tratamento farmacológico, mas também à conscientização do paciente a respeito da patologia ${ }^{27}$. Além do tratamento farmacológico, são recomendadas modificaçóes no estilo de vida e prática de hábitos saudáveis, tais como a adoçẫo de uma alimentação equilibrada e prática regular de atividade física, objetivando o tratamento e prevenção da HAS ${ }^{1}$.

Baludíno e Jacopetti utilizaram WHOQOL-bref em seu estudo e observaram resultados semelhantes aos 
deste. Em seu estudo, verificou-se que, tanto para o gênero feminino como para o masculino, a maior variação das respostas ocorreu no domínio físico que se refere à dor e ao desconforto, energia e fadiga, sono e repouso, mobilidade, atividade da vida cotidiana, dependência de medicação ou tratamento, e capacidade de trabalho. Sendo a menor variação das respostas correspondente ao domínio psicológico, que se referem os sentimentos positivos e negativos, memória e concentração, autoestima, imagem corporal e aparência, religião e crenças ${ }^{28}$.

Verificou-se neste artigo que o domínio com menor pontuação foi o físico, composto pelas seguintes facetas: dor e desconforto, energia, sono, mobilidade, atividades da vida cotidiana e dependência de medicações, o que pode ser atribuído à própria HAS. Em contraponto, no trabalho desenvolvido por França et al., o domínio que apresentou menor pontuação foi o meio ambiente, sendo este composto por facetas como: segurança, educação, lazer, moradia, acesso aos serviços de saúde e transporte, cuja satisfação depende da capacidade financeira $^{29}$. Dado também observado no estudo realizado por Tavares et al., no qual identificou-se menor escore no domínio de meio ambiente em indivíduos hiperten$\operatorname{sos}^{30}$. Outro estudo, por sua vez, realizado com idosos com doenças crônicas, observou menor escore para o referido domínio entre aqueles com morbidade crônica ${ }^{31}$.

Balduíno e Jacopetti demonstraram que a maioria dos indivíduos sentia-se satisfeita com a sua qualidade de vida, porque adotam hábitos considerados saudáveis, embora precisem de algum tipo fármaco para isso ${ }^{28}$. Neste estudo verificou-se maior escore de qualidade de vida nos indivíduos não idosos, dado semelhante ao observado por Balduíno e Jacopetti.

O trabalho de Mazo et al. ${ }^{38}$ observou a prática de atividade física e qualidade de vida de mulheres idosas. Naquele trabalho, ao relacionar o domínio físico com o nível de atividade física, constatou-se que as idosas mais ativas tinham energia suficiente para o dia a dia, boa capacidade de locomoção, satisfação com o seu sono e com a sua capacidade para exercer as atividades de vida diária e de trabalho. Quanto ao nível de atividade física e à sua relação com o domínio psicológico, constatou-se que as idosas mais ativas estáo satisfeitas com a sua autoimagem e a autoestima, e consideram que a vida tem bastante sentido. $\mathrm{O}$ domínio das relaçôes sociais não se mostrou associado com o nível de atividade física. Contudo, o ato de praticar atividade física por parte da idosa era visto com bons olhos pela família e amigos, produzindo na idosa bem estar e melhora na autoestima. Do mesmo modo, o domínio meio ambiente não apresentou diferença significativa em relação ao nível de atividade física, porém foi percebido que a prática de atividade física pelas idosas foi influenciada pelo ambiente, principalmente nos aspectos de segurança física, recursos financeiros, informação, oportunidade de lazer e transporte, entre outros. Quanto à percepção das idosas sobre sua qualidade de vida de modo geral, foi observado que aquelas com elevado nível de escolaridade, pertencentes à classe econômica mais alta e com percepção positiva de sua saúde expressaram ter boa qualidade de vida. Diferentemente deste trabalho, em que foi comparado idosos e não-idosos, mostrando que a idade é um fator que afeta sim a qualidade de vida quando mais avançada.

Em relação à idade, Irigaray e Schneider destacaram que, em média, as mulheres vivem cerca de cinco a seis anos a mais do que os homens, e essa longevidade é concedida a tendência ao autocuidado e a busca de assistência médica durante toda a vida. As mulheres tendem a ter problemas de saúde de longa duração, crônicos e incapacitantes; já os homens tendem a desenvolver doenças fatais de curta duraçáa ${ }^{32}$. Da mesma forma, no estudo realizado por Borim et al., averiguou-se que a maior parte da amostra foi constituída por $76 \%$ de mulheres, sendo esse dado interpretado como parte do processo de feminização da velhice ${ }^{33}$. Pucci et al. observaram uma prevalência de $71,9 \%$ de mulheres e Tavares et al. observaram $61 \%$ de mulheres em suas pesquisas ${ }^{30,34}$. Dado também observado neste estudo, onde se verificou uma prevalência de $64,7 \%$ de pacientes do gênero feminino.

Sabe-se que o avançar da idade intensifica o risco para as doenças crônicas, ocasionando o aumento no consumo contínuo de fármacos, além da diminuição da capacidade funcional, tornando-se um risco para uma menor qualidade de vida ${ }^{35,36}$. De acordo com Lalonde et al., o estilo de vida pode ser decisivo no processo saúde-doença, no qual verificaram a dependência direta das condiçôes dos indivíduos durante o decorrer da vida, influenciando na saúde, o que poderá resultar no menor ou maior uso fármacos ${ }^{37}$.

É imprescindível destacar que este estudo apresenta algumas limitações que impedem maiores generalizaçóes, visto que a validade externa tornase comprometida pelo estudo ser realizado com uma amostra de pacientes hipertensos acompanhados em uma única UBS. Ademais, não é uma pesquisa longitudinal, o que não possibilita identificar associaçóes causais.

\section{Conclusão}

Conforme os resultados indicam, os indivíduos apresentaram predominantemente excesso de peso corpóreo e risco de desenvolvimento de doenças cardiovasculares. O gênero masculino associou-se aos maiores escores nos domínios psicológicos e de meio ambiente. O domínio de meio ambiente apresentou 
associação positiva com a $\mathrm{CMB}$ e associação negativa com a idade dos indivíduos. Destaca-se ainda que a qualidade de vida tornou-se menor com o aumento da idade, pois os indivíduos não idosos apresentaram maior escore total.

\section{Referências}

1. Sociedade Brasileira de Cardiologia/Sociedade Brasileira de Hipertensão/Sociedade Brasileira de Nefrologia. VI Diretrizes Brasileiras de Hipertensão. Arq Bras Cardiol. 2010;95(1):1-51.

2. World Health Organization. World Health Statistics 2012. Geneva: World Health Organization, 2012.

3. Ferreira SRG, Zanella MT. Epidemiologia da hipertensão arterial associada à obesidade. Rev Bras Hipert. 2000;2(7):128-35.

4. Sales CM, Tamaki EM. Adesão às medidas de controle da hipertensão arterial sistêmica: o comportamento do hipertenso. Cogit Enfer. 2007;12(2):157-63.

5. Noblat ACB, Lopes MB, Lopes GB, Lopes AA. Complicaçōes da hipertensão arterial em homens e mulheres atendidos em um ambulatório de referência. Arq Bras Cardiol. 2004;83(4):308-13.

6. Pessuto J, Carvalho EC. Fatores de risco em indivíduos com hipertensão arterial. Rev Latino-Am Enfermagem. 1998;6(1):33-9.

7. Vilarta R. Diagnóstico da alimentação saudável e atividade física para a qualidade de vida. Campinas: IPES; 2007.

8. Fleck MPA, Leal OF, Louzada S, Xavier M, Chachamovich E, Vieira $G$, et al. Aplicação da versão em português do instrumento da avaliação de qualidade de vida da Organização Mundial da Saúde (WHOQOL-100). Rev Saúde Pública. 1999;33(2):198-205.

9. Fleck MPA, Leal OF, Louzada S, Xavier M, Chachamovich E, Vieira $G$ et al. Aplicação da versão em português do instrumento abreviado de avaliação da qualidade de vida "WHOQOL-bref". Rev Saúde Pública. 2000;34(2):178-83.

10. Sampaio LR, Figueiredo VC. Correlação entre o índice de massa corporal e os indicadores antropométricos de distribuição de gordura corporal em adultos. Rev Nutr. 2005;18(1):53-61.

11. Ring BL. Guidelines for diagnosis and treatment of high cholesterol. JAMA. 2001;286(19): 2401-2.

12. Blackburn GL, Thornton PA. Nutritional assessment of the hospitalized patients. Med Clin North Am. 1979;63(5):1103-15.

13. Paiva SAR, Godoy I, Padovani CR, Geraldo RRC, Campana AO. O uso das pregas cutâneas e da circunferência muscular do braço no diagnóstico de desnutrição energético-proteica em pacientes adultos: estudo crítico. Rev Hosp Clin Fac Med. 1992;47(5):223-30.
14. World Health Organization. Obesity: preventing and managing the global epidemic: report of a WHO consultation on obesity. Geneva: World Health Organization, 1998.

15. Lipschitz DA. Screening for nutritional status in the elderly. Prim Care. 1994;21(1):55-67.

16. Souza ARA, Costa A, Nakamura D, Mocheti LN, Stevanato PRF, Ovandosouza LA. Um estudo sobre hipertensão arterial sistêmica na cidade de Campo Grande, MS. Arq Bras Cardiol. 2007;88(4):441-46.

17. Helvaci MR, Kaya H, Alcin A, Kuvandik G. Prevalence of white coat hypertension in underweight and overweight subjects. Int Heart J. 2007;48:605-13.

18. O’Donnell CJ, Elousa R. Cardiovascular risk factors - insights from Framingham Heart Study. Rev Esp Cardiol. 2008;61(3):299-310.

19. Fernandes RA, Nogueira A, Christofaro DGD. Utilização do índice de massa corporal e dobra cutânea tricipital como indicadores de adiposidade corporal. Rev Educ Fís. 2007;18(1):1-7.

20. Silva PC, Zaffari D. Prevalência de excesso de peso e associação com outras variáveis em indivíduos adultos atendidos em unidade básica de saúde. Scientia Medica. 2009;19(1):17-26.

21. Melchiors AC, Correr CJ, Pontarolo R, Santos FLS, Souza RAP. Qualidade de vida em pacientes hipertensos e validade concorrente do Minichal-Brasil. Arq Bras Cardiol. 2010;94(3):357-64.

22. Figueiredo NN, Asakura L. Adesão ao tratamento anti-hipertensivo: dificuldades relatadas por indivíduos hipertensos. Acta Paul Enferm. 2010;23(6):782-7.

23. Carneiro G, Faria NA, Filho FFR, Guimarães DL, Ferreira SRG, Zanella MT. Influência da distribuição da gordura corporal sobre a prevalência de hipertensão arterial e outros fatores de risco cardiovascular em indivíduos obesos. Rev Assoc Med Bras. 2003;49(3):306-11.

24. Barbosa LS, Scala LCN, Ferreira MG. Associação entre marcadores antropométricos de adiposidade corporal e hipertensão arterial na população adulta de Cuiabá, Mato Grosso. Rev Bras Epidemiol. 2009;12(2):237-47.

25. Souza LJ, Neto CG, Chalita FEB, Reis AFF, Bastos DA, Filho JTDS et al. Prevalência de Obesidade e Fatores de Risco Cardiovascular em Campos, Rio de Janeiro. Arq Bras Endocrinol Metab. 2003;47(6):669-76.

26. Lima J, Nóbrega L, Nóbrega M, Bandeira F, Souza AGP. Dislipidemia pós-prandial como achado precoce em indivíduos com baixo risco cardiovascular. Arq Bras Endocrinol Metab. 2002;46(3):249-54.

27. Li W, Liu L, Puente JG, Li Y, Jiang X, Jin S et al. Hypertension and health-related quality of life: an epidemiological study in patients attending hospital clinics in China. J Hypertens. 2005;23(9):1667-76.

28. Balduíno E, Jacopetti SR. Levantamento da qualidade de vida de um grupo de idosos. Bol Enf. 2009;3(2):31-47.

29. França ISX, Coura AS, França EG, Basílio NNV, Souto 
RQ. Qualidade de vida de adultos com lesão medular: um estudo com WHOQOL-bref. Rev Esc Enferm. 2011;45(6):1364-71.

30. Tavares DMS, Martins NPF, Dias FA, Diniz MA. Qualidade de vida de idosos com e sem hipertensão arterial. Rev Eletr Enf. 2011;13(2):211-8.

31. Nóbrega TCM, Jaluu O, Machado NA, Paschoal SMP, Jacob Filho W. Quality of life and multimorbidity of elderly outpatients. Clinics. 2009;64(1):45-50.

32. Iragaray TQ, Schneider RH. Dimensóes de personalidade, Qualidade de vida e depressão em idosas. Psicol em Estudo. 2009;14(4):759-66.

33. Borim FSA, Guariento MA, Almeida EA. Perfil de adultos e idosos hipertensos em unidade básica de saúde. Rev Bras Clin Med. 2011;9(2):107-11.

34. Pucci N, Pereira MR, Vinholes DB, Pucci P, Campos ND. Conhecimento sobre Hipertensão Arterial Sistêmica e
Adesão ao Tratamento Anti-Hipertensivo em Idosos. Rev Bras Cardiol. 2012;25(4):322-9.

35. Minayo MCS, Hartz ZMA, Buss PM. Qualidade de vida e saúde: um debate necessário. Ciên Saúde Coletiva. 2000;5(1):7-18.

36. Kusumoto L, Marques S, Haas VJ, Rodrigues RAP. Adultos e idosos em hemodiálise: avaliação da qualidade de vida relacionada à saúde. Acta Paul Enferm. 2008;5(21):152-9.

37. Lalonde L, O'Connor A, Joseph L, Grover SA. Health-related quality of life in cardiac patients with dyslipidemia and hypertension. Qual Life Res. 2004;4(13):793-804.

38. Mazo GZ, Mota JAPS, Gonçalves LHT. Atividade física e qualidade de vida de mulheres idosas. RBCEH - Revista Brasileira de Ciências do Envelhecimento Humano. 2005;1:115-8.

\section{Como citar este artigo:}

Kruger AP, Mergener M, Pozzobon A, Moreira TR. Avaliação da qualidade de vida de hipertensos usuários de uma unidade básica de saúde. Rev. Aten. Saúde. 2015;13(46):43-50 